\title{
FIR-spectroscopy of the FI-SDW excitation spectrum in a quasi-1D organic conductor
}

\author{
A.M. Gerrits ${ }^{a}$, T.J.B.M. Janssen ${ }^{a}$, A.S. Perel ${ }^{\text {b }, ~ J . S . ~ B r o o k s ~}{ }^{b}$, A. Wittlin ${ }^{a}$, \\ J.A.A.J. Perenboom ${ }^{a}$, and P.J.M. van Bentum ${ }^{a}$ \\ ${ }^{a}$ High Field Magnet Laboratory and Research Institute for Materials, \\ University of Nijmegen, NL-6525 ED Nijmegen, The Netherlands \\ ${ }^{\mathrm{b}}$ Department of Physics, Boston University and F. B. National Magnet Laboratory, \\ M.I.T, Cambridge, Massachusetts 02139, USA.
}

\begin{abstract}
The anisotropic quasi one-dimensional conductor (TMTSF $)_{2} \mathrm{X}\left(\mathrm{X}=\mathrm{ClO}_{4}, \mathrm{PF}_{6}\right)$ exhibits a cascade of field-induced spin density wave (FI-SDW) phase transitions. In this contribution, we present a magneto-optical study of the far infrared excitation spectrum of (TMTSF) ${ }_{2} \mathrm{ClO}_{4}$ as a function of magnetic field and temperature covering the low field part of the phase diagram of this system.

The observed transition temperatures of the various FI-SDW subphases follow closely the established phase diagram. Our results show that the magnitude of the SDW gap depends neither on magnetic field nor on temperature. In addition we find evidence for the existence of a collective mode excitation in the SDW state, indicating a relatively strong pinning to the lattice. Further indication of a large electron lattice coupling is given by the strong phonon renormalisation in the SDW state.
\end{abstract}

\section{Introduction}

Conductors based on the organic molecule tetramethyltetraselenafulvalene (TMTSF) form an interesting class of low dimensional materials. The conductivity occurs mainly along the one-dimensional chains but the conductivity in the $b$-direction perpendicular to the chains is only about one order of magnitude less. The corresponding Fermi surface consists of two slightly warped planes and is sensitive to a nesting instability. This leads to a Peierls transition at low temperatures into a spin-density wave ground state for this class of materials.

The compound (TMTSF) ${ }_{2} \mathrm{ClO}_{4}$ is exceptional in the sense that it remains metallic down to liquid helium temperatures and becomes superconducting below $1.2 \mathrm{~K}$. In a magnetic field perpendicular to the conducting plane this system shows a series of highly unusual phase transitions to a SDW groundstate. These phase transitions are quite prominent in both the transport and the thermodynamic properties. The first qualitative explanation of the FI-SDW phases was given by Gor'kov and Lebed. In a subsequent theoretical treatment to explain the various experimental anomalies observed, Montambaux and Poilblanc assumed a constant carrier density in the various FISDW phases and using a mean field approach concluded that a multiplet of gaps would form separating Landau like bands[1]. The largest of these gaps would be at the Fermi level and its size would roughly scale as the FI-SDW transition temperature $[1,2]$.

Because infrared spectroscopy is a very direct method to observe gap formation in the electronic density of states, we investigated the excitation spectrum of (TMTSF) ${ }_{2} \mathrm{ClO}_{4}$ between $2 \mathrm{~cm}^{-1}$ and $45 \mathrm{~cm}^{-1}$ in fields up to $20 \mathrm{~T}$ and at temperatures ranging from $0.4 \mathrm{~K}$ to $6.0 \mathrm{~K}$.

\section{Experimental details}

The samples used were arranged in a gridlike structure with the $a b$ plane of the crystals coinciding with the plane of the grid[3]. The samples were then placed in a ${ }^{3} \mathrm{He}$ cooled sample holder and mounted in the bore of either a $18 \mathrm{~T}$ superconducting magnet or of a $20 \mathrm{~T}$ Bitter magnet. The sample was cooled at a rate of $30 \mathrm{mK} / \mathrm{min}$ through the anion ordering temperature at $24 \mathrm{~K}$. The magnetic field was applied along the $c$-axis, perpendicular to the grid. We used various FIR setups to cover the spectral range from about $2 \mathrm{~cm}^{-1}$ to $45 \mathrm{~cm}^{-1}$ : an optically pumped molecular FIR laser, a microwave harmonic generator and two wide band Fourier spectrometers (Bruker IFS 113v and Grubb Parsons). The spectra obtained with the various sources are overlapping in frequency range and are in good agreement with each other. For the grid geometry employed, the transmitted power in the spectral region of interest is polarized perpendicularly to the grid and will depend mainly on the conductivity $\sigma(\omega)$ along the $b$-axis. This was confirmed in separate measurements using polarised incident radiation.

In the geometry used one is quite sensitive to small changes in the excitation spectrum. A drawback, unavoidable however for these small needle like crystals, 


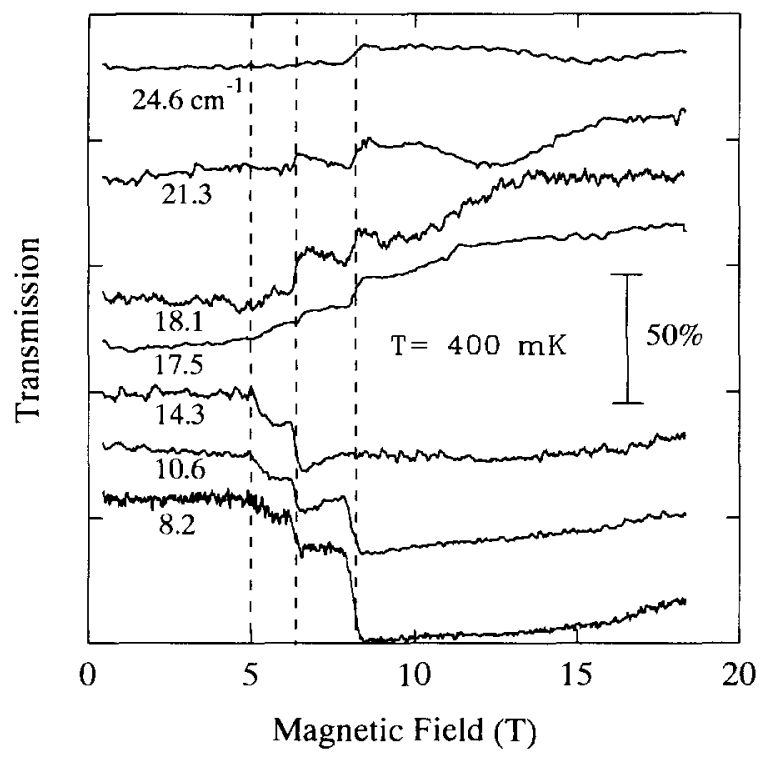

Figure 1. Transmission versus magnetic field for monochromatic energies from $8.2 \mathrm{~cm}^{-1}$ to $24.6 \mathrm{~cm}^{-1}$. The dashed lines indicate the transition fields as determined from simultaneous magnetoresistance measurements on a crystal mounted in the vicinity of the sample grid but carefully shielded from radiation.

is that it is very difficult to obtain absolute values for $\sigma(\omega)$ using a Kramers Kronig type of analysis. After the measurements we evaporated a gold film on the sample grid to obtain reference spectra for normalization purposes.

\section{Spectroscopic results and discussion}

Figure 1 presents the transmission through the sample grid versus magnetic field for several monochromatic energies generated by the FIR laser[3]. The transmission is normalised on the transmission in zero field. We find stepwize changes in the transmission as the sample enters the different FI-SDW phases with nearly field independent plateaux in between. Because the spectra showed large hysteris in the highest transition field, depending on the sweep direction, only traces recorded with increasing field are shown. The most interesting aspect of these traces at monochromatic energies is that the changes near the phase transitions are quasi-steplike, reminiscent of the steps in the Hall voltage. At low frequencies, the sample reflectivity (proportional to the transmitted signal) decreases with increasing field. It would correspond with a smaller conductivity, in agreement with the opening of a (partial) gap in the excitation spectrum. At frequencies above $14 \mathrm{~cm}^{-1}$ the signal increases at the SDW transitions, as one would expect when (part of) the single particle density of states piles up above the gap edge.

Figure 2 shows the FIR transmission through the grid as a function of the applied magnetic field at various indicated temperatures[4]. A FIR energy of $8.3 \mathrm{~cm}^{-1}$ was used. With increasing temperature we find the FI-SDW phase transitions that can be resolved to decrease in number and to shift to higher fields. At low temperatures the transitions occur almost discontinuously within a very narrow field range, indicative of a first order nature of the phase transitions. At higher temperatures the transitions are broadened and the corresponding changes in the FIR transmission become smaller. For the purpose of constructing a phase diagram, we have chosen the midpoint of the steps in the transmission signal as the transition field at each temperature. The points thus acquired are plotted in the right part of figure 2, together with the specific heat data of Fortune et al. [5]. The overall agreement is quite good, giving further confidence that we are indeed optically probing the SDW formation.

The complete energy spectrum versus magnetic field is shown in figure 3 . The spectra are normalised to a zero field spectrum. The data from the monochromatic energies of figure 1 have been added as symbols. Within one SDW phase, the spectra were virtually identical (corresponding to a flat region in the monochromatic data) so we plotted only three spectra labeled $\mathrm{N}=2,1,0$ taken at fields of $6,7.5$, and 8 T. From figure 3 several features can be identified: (a) a large signal increase is found, starting at about $14 \mathrm{~cm}^{-1}$ in all FISDW-phases. (b) Sharp features are found at 17 and $32 \mathrm{~cm}^{-1}$, and futhermore, (c) a resonance like peak at $2.5 \mathrm{~cm}^{-1}$. All features grow more pronounced for higher fields or equivalently lower FISDW-label None of them tend to shift in energy for different magnetic fields.

The main feature in the spectra is the opening of a gap below $14 \mathrm{~cm}^{-1}$. In contrast to the prediction in Ref.[1], no multiple gaps are observed and, surprisingly, the fundamental gap appears to be field independent. However, the signal strength in the gap is not zero but does depend on the subphase index. We must conclude that the gap is not uniform in $k$-space.

To explain the results, we propose the following qualitative and simple model: As the field increases, the system will become more sensitive to a nesting instability until part of the charge carriers condense into a collective SDW groundstate. It will now take a finite amount of energy (the gap) to create a free electron excitation. The free energy of the system was shown to be lowest if the area of the normal carrier pockets on the Fermi surface is quantized[6]. The total gapped area, or equivalently the density of electrons in the collective state will increase discontinuously upon each phase transition. These discontinous changes agree with the general observation of an increase of the magnetoresistance and a stepwise increase of the Hall voltage.

At $17 \mathrm{~cm}^{-1}$ and $32 \mathrm{~cm}^{-1}$ phonon like features are seen. The $32 \mathrm{~cm}^{-1}$ feature is basically an acoustic phonon mode which becomes FIR active due to zone folding caused by the $\mathrm{ClO}_{4}$ anion ordering tran- 


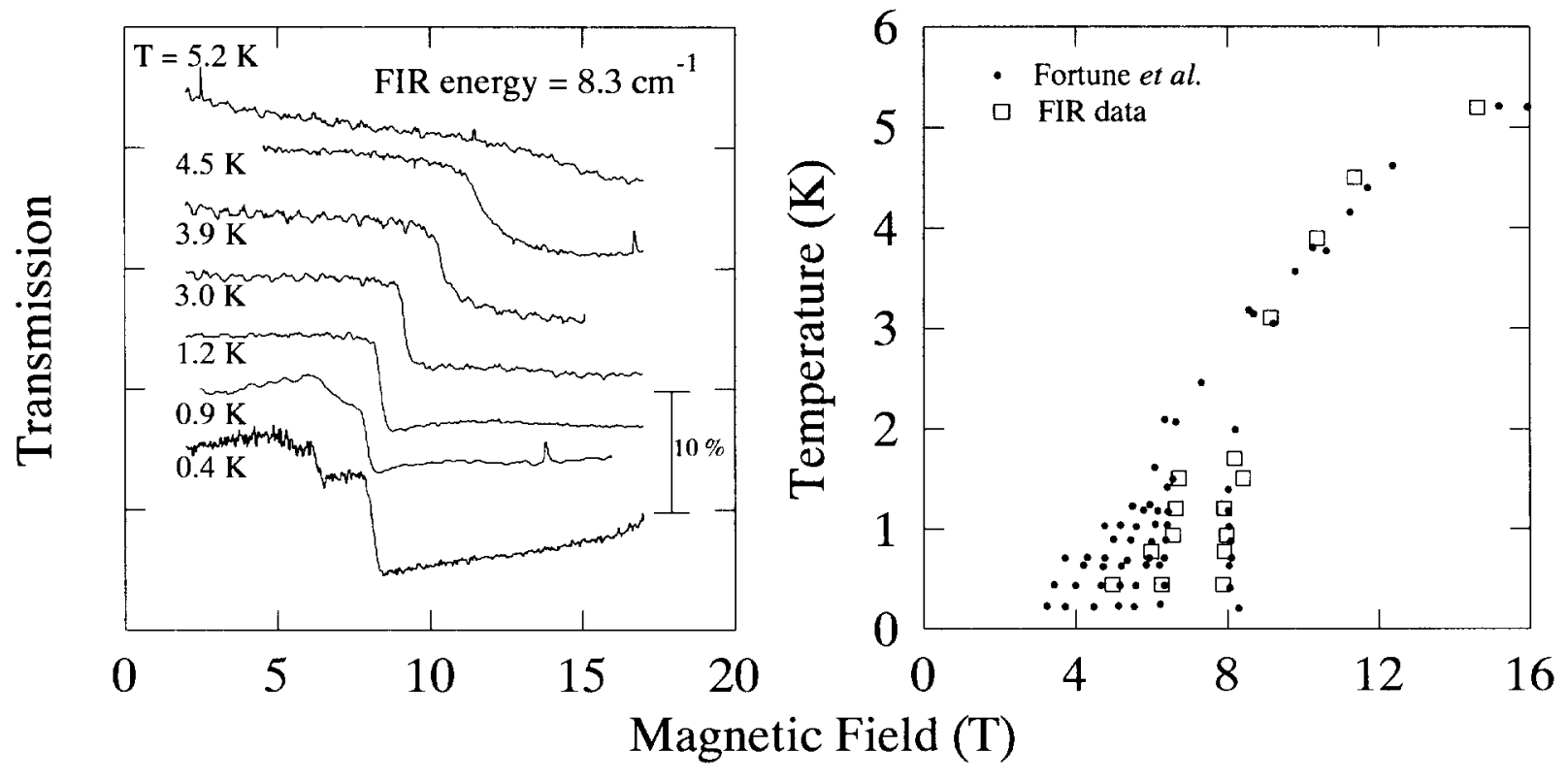

Figure 2. Left: FIR signal well within the SDW gap as a function of the applied magnetic field at different temperatures. The traces have been offset for clarity. Right: Phase diagram deduced from the transitions observed optically. The small dots indicate the corresponding results from specific heat measurements, Ref. 5 .

sition[7]; the $17 \mathrm{~cm}^{-1}$ feature is presumably of similar origin. When the spectra in the metallic state are normalized to the reference spectra taken on the goldcovered sample both features appear as clear peaks in the spectra. Upon increasing the magnetic field into the SDW states, we find a strong change in the phonon lineshape. This could be due to the increased coupling to electronic excitations just above the SDW gap. These changes, although unexpected for SDW systems, are in themselves not very surprising given the additional incommensurate modulation which breaks translational symmetry and changes the selection rules for optically active $(k=0)$ phonon modes. However, it must be noted that both phonons are also present in the metallic state and the effect of the SDW formation is primarily a change in lineshape.

In order to investigate the temperature dependence of the gap feature we measured the energy spectrum as a function of temperature at a magnetic field of $10 \mathrm{~T}$ with a slow scan Fourier spectrometer optimized in the long wavelength region (figure 4). At this field we probe only the high field $(\mathrm{N}=0)$ phase.

We observe that the gaplike structure below $14 \mathrm{~cm}^{-1}$ does not shift to lower energies with increasing temperature, as one would expect in a mean field picture. Instead it remains at a fixed position. The signal strength inside the gap remains nearly constant with increasing temperature until the transition temperature is reached. This is illustrated more clearly in the inset of figure 4 which shows the transmission at $9.5 \mathrm{~cm}^{-1}$ as a function of temperature. The sudden change is indicative of a first order phase transition. A similar sharp decrease of the order parameter was found in the $\mu \mathrm{SR}$ data by Le et al.[8]. Thus, we find that both the magnetic field dependence and the tem-

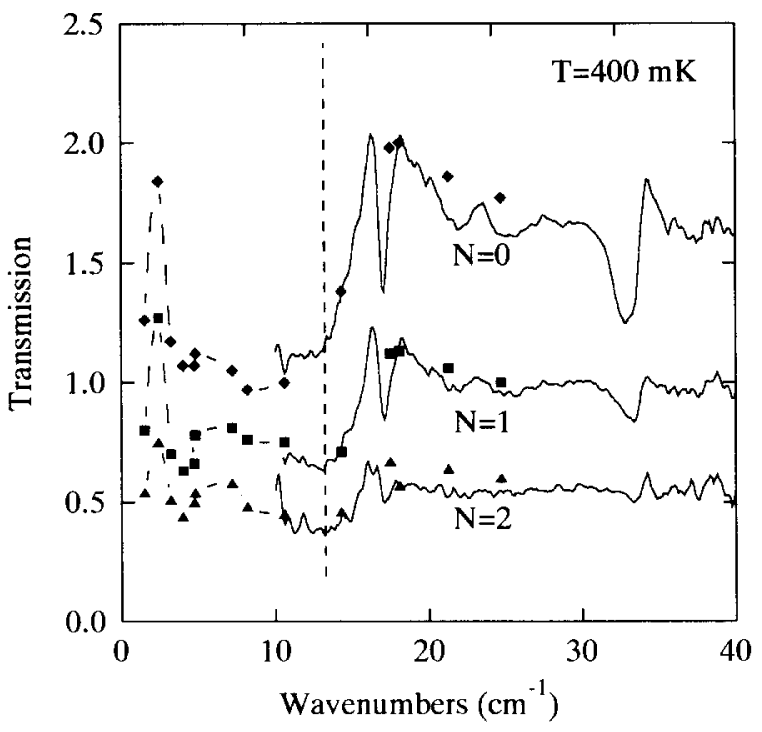

Figure 3. Normalised spectra for three different magnetic fields corresponding to the $N=0,1,2$ FI-SDW-subphases. The data collected for monochromatic energies are shown as points. The upper and lower trace have been offset for clarity by, respectively, +0.5 and -0.5 . 
perature dependence of the SDW gap are quite different from a traditional BCS-like mean field prediction.

The fact that, above $T_{c}$, shallow remnants of the gap structure remain visible is also unexpected in a mean field model and indicates possibly the presence of SDW fluctuations with no long range magnetic order.

In order to check the assignment of the $14 \mathrm{~cm}^{-1}$ edge to the SDW gap we performed FIR measurements on a similar grid of (TMTSF) ${ }_{2} \mathrm{PF}_{6}$ crystals, which also show a SDW transition, however not field induced. Below the SDW ordering temperature which is $12 \mathrm{~K}$ at ambient pressure for this material, a decrease of the transmission signal below $32 \mathrm{~cm}^{-1}$ is observed. Near this edge we find no visible phonon structure in the spectra, due to zone folding because there is no anion ordering in the $\mathrm{PF}_{6}$ compound. Assigning this edge to the opening of the SDW gap, we find that the energy of this gap is about twice as large as the sharp edge in the (TMTSF) ${ }_{2} \mathrm{ClO}_{4}$ spectra, in accordance with the twice as large value of $\mathrm{T}_{\mathrm{c}}$ in (TMTSF) ${ }_{2} \mathrm{PF}_{6}$.

Further confirmation of our observations was given recently by Lefebvre et al.[9] who performed far infrared photoconductivity (PC) measurements on (TMTSF) ${ }_{2} \mathrm{ClO}_{4}$ in the FI-SDW regime. Their results show a large $P C$ peak at $19 \mathrm{~cm}^{-1}$ (stretched from 14 to $20 \mathrm{~cm}^{-1}$ ) caused by resonant photon absorption near the SDW gap. The PC peak does not shift as a function of field or temperature.

Sofar we have not mentioned the very low frequency mode which is visible in figure 3 and 4 at about $2.5 \mathrm{~cm}^{-1}$. This mode does not shift with field and slightly hardens when going to lower temperatures. Although the relative accuracy decreases near the extreme low frequency end of the spectrum in figure 4, it is clear that the intensity of this mode is weakly temperature dependent until it vanishes near the critical temperature. This is in agreement with the assignment to a collective mode excitation of the SDW state, where the hardening at low temperatures is due to a more effective pinning of the condensed state.

\section{Conclusion}

In conclusion, we present spectroscopic data on the temperature dependence of the FI-SDW formation in (TMTSF $)_{2} \mathrm{ClO}_{4}$. The optically detected phase transitions follow closely the phase diagram inferred from thermodynamic measurements. The value of the SDW gap is relatively insensitive to both magnetic field and temperature. The field transitions at low temperatures are of first order. The remains of some shallow gap structure in the spectra above $T_{c}$ indicate possible fluctuations of the FI-SDW state. We find evidence for the presence of a collective mode near $2.5 \mathrm{~cm}^{-1}$ which is weakly temperature dependent, indicating possible depinning at higher temperatures.

We thank Paul Chaikin and coworkers at Princeton University for providing the samples.

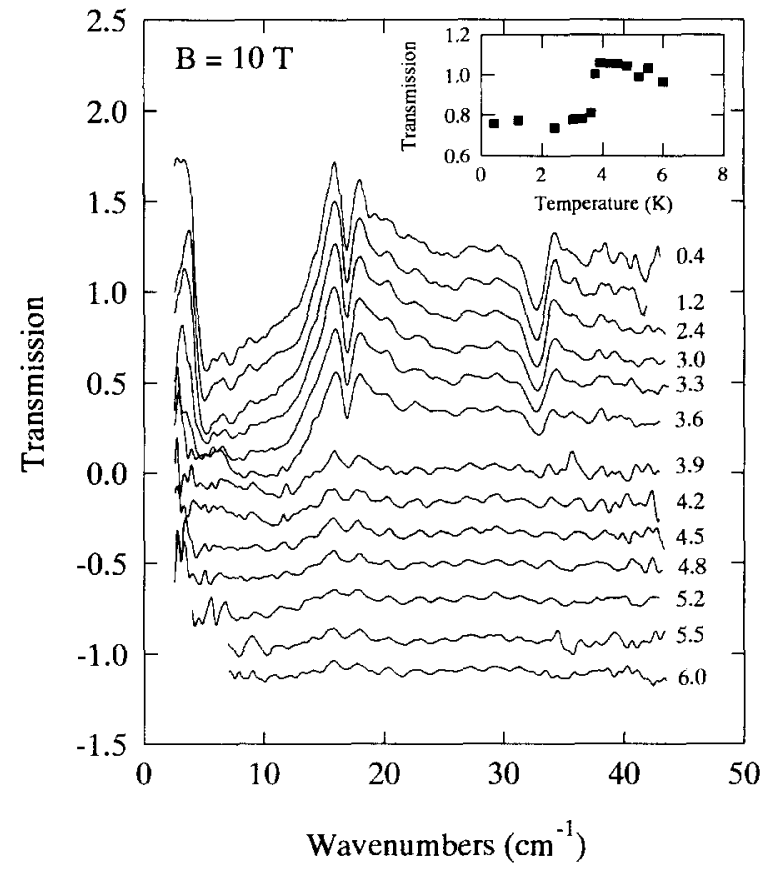

Figure 4. Normalized transmission, as a function of frequency at various temperatures. The spectra are offset for clarity.

\section{REFERENCES}

1. G. Montambaux, and D. Poilblanc, Phys. Rev. B 37, 1913 (1988).

2. G. Montambaux, M.J. Naughton, R.V. Chamberlin, X. Yan, P.M. Chaikin, and M.Y. Azbel, Phys. Rev. B 39885 (1989)

3. T.J.B.M. Janssen, A.S. Perel, A.M. Gerrits, W. Kang, J.S. Brooks, A. Wittlin, J.A.A.J. Perenboom, and P.J.M. van Bentum, Phys. Rev. B 46, 8663 (1992).

4. A.M. Gerrits, T.J.B.M. Janssen, J.S. Brooks, A. Wittlin, J.A.A.J. Perenboom, and P.J.M. van Bentum, J. Phys. IV (France) Colloq. C2-3, 299 (1993).

5. N.A. Fortune, J.S. Brooks, M.J. Graf, G. Montambaux, L.Y. Chaing, J.A.A.J. Perenboom, and D. Althof, Phys. Rev. Lett. 64, 621 (1988).

6. L.P Gor'kov, and A.G. Lebed', J. Phys. (Paris) Lett. 45, L433 (1984).

7. J.E. Eldridge, C.C. Homes, F.E. Bates, and G.S. Bates, Phys. Rev. B 325156 (1985).

8. L.P. Le, A. Keren, G.M. Luke, B.J. Sternlieb, W.D. Wu, Y.J. Uemura, J.H. Brewer, T.M. Riseman, R.V. Upasani, L.Y. Chiang, W. Kang, P.M. Chaikin, T. Csiba, and G. Grüner, Phys. Rev. B 487284 (1993).

9. J. Lefebvre, J. Beerens, C. Bourbonnais, L.G. Caron, C. Lenoir, and P. Batail, Phys. Rev. Lett. 72, 3417 (1994) 